Supporting Information for:

\title{
Dimethylammonium Trichlorocuprate(II): Structural Transition, Low- Temperature Crystal Structure, and Unusual Two-Magnetic Chain Structure Dictated by Non-bonding Chloride-Chloride Contacts
}

\author{
Roger D. Willett* \\ Department of Chemistry, Washington State University, Pullman, Washington 99164 \\ Brendan Twamley \\ University Research Office, University of Idaho, Moscow, ID 83844 \\ Wouter Montfrooij ${ }^{\dagger}$, Garrett G. Granroth, and Stephen E. Nagler \\ Oak Ridge National Laboratory, Oak Ridge, Tennessee 37831 \\ Donavan W. Hall \\ National High Magnetic Field Laboratory, Florida State University, Tallahassee, Florida 32210 \\ Ju-Hyun Park, Brian C. Watson ${ }^{\S}$, and Mark W. Meisel \\ Department of Physics, University of Florida, Gainesville, Florida 32611-8440 \\ Daniel R. Talham* \\ Department of Chemistry, University of Florida, Gainesville, Florida 32611-72000
}

Contents: Complete scans of Figure 1 powder neutron data. 


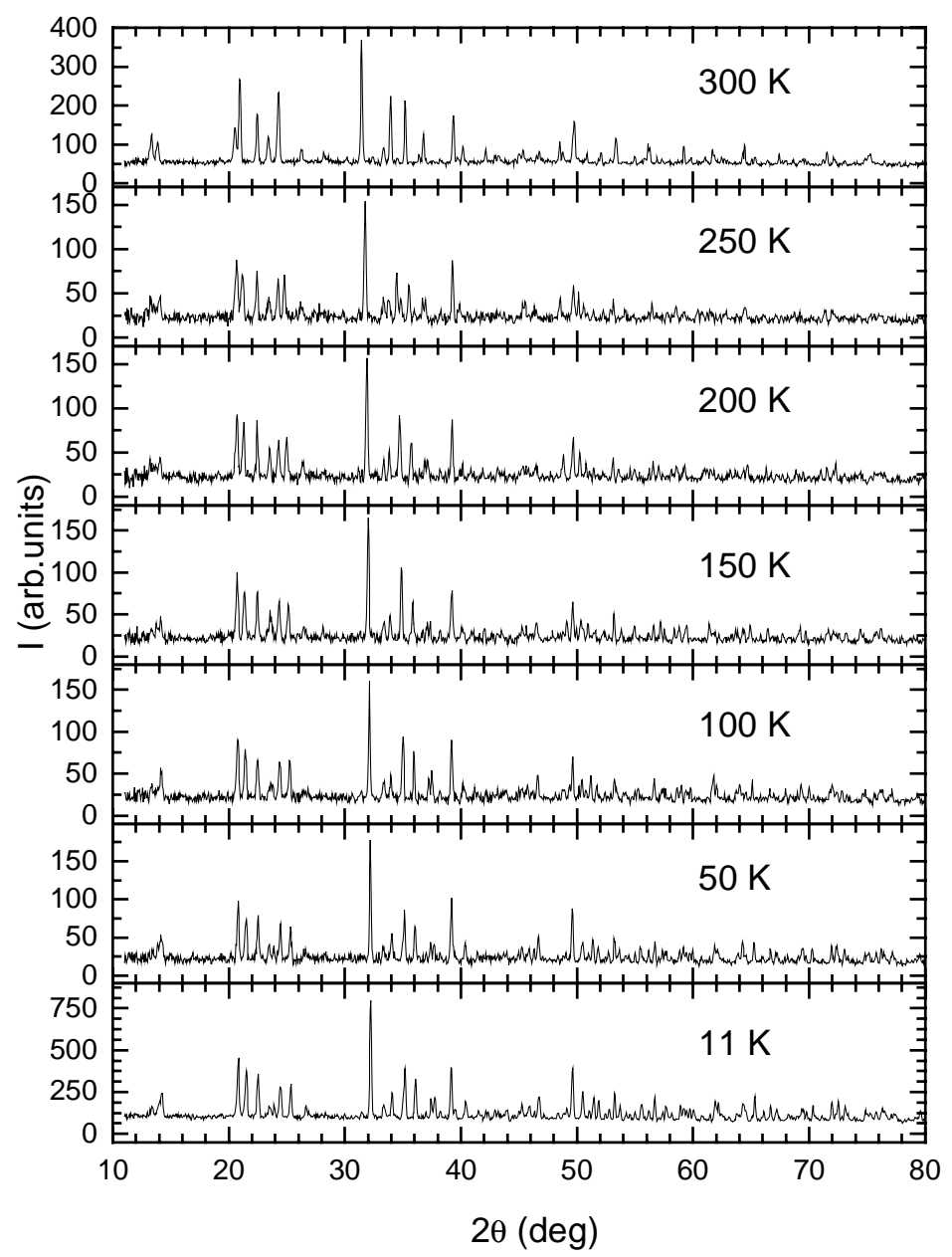

Supporting Figure 1. The intensity of the powder diffraction experiment are shown as a function of $2 \theta$ and temperature. When cooling from $300 \mathrm{~K}$ to $250 \mathrm{~K}$, the peaks near $21^{\circ}$ and $33^{\circ}$ split. Additional changes are observed between $100 \mathrm{~K}$ and $150 \mathrm{~K}$, but no additional evolution of the spectra was observed between $11 \mathrm{~K}$ and 50 $\mathrm{K}$. The sample consisted of $1.1 \mathrm{~g}$ of randomly oriented deuterated microcrystals. 